\title{
Maternal cardiac diseases and pregnancy: maternal, obstetrical and neonatal outcomes: a systematic literature review Bonnet V. ${ }^{1}$, Simonet T. ${ }^{1}$, Labombarda F. ${ }^{2}$, Dreyfus M. ${ }^{3}$, Hanouz J.-L. ${ }^{1}$
}

1University Hospital Caen, Dept of Anaesthesiology \& Intensive Care, Caen, France, 2University Hospital Caen, Department of Cardiology, Caen, France, 3University Hospital Caen, Department of Gynaecology, Obstetric and Reproductive Medicine, Caen, France

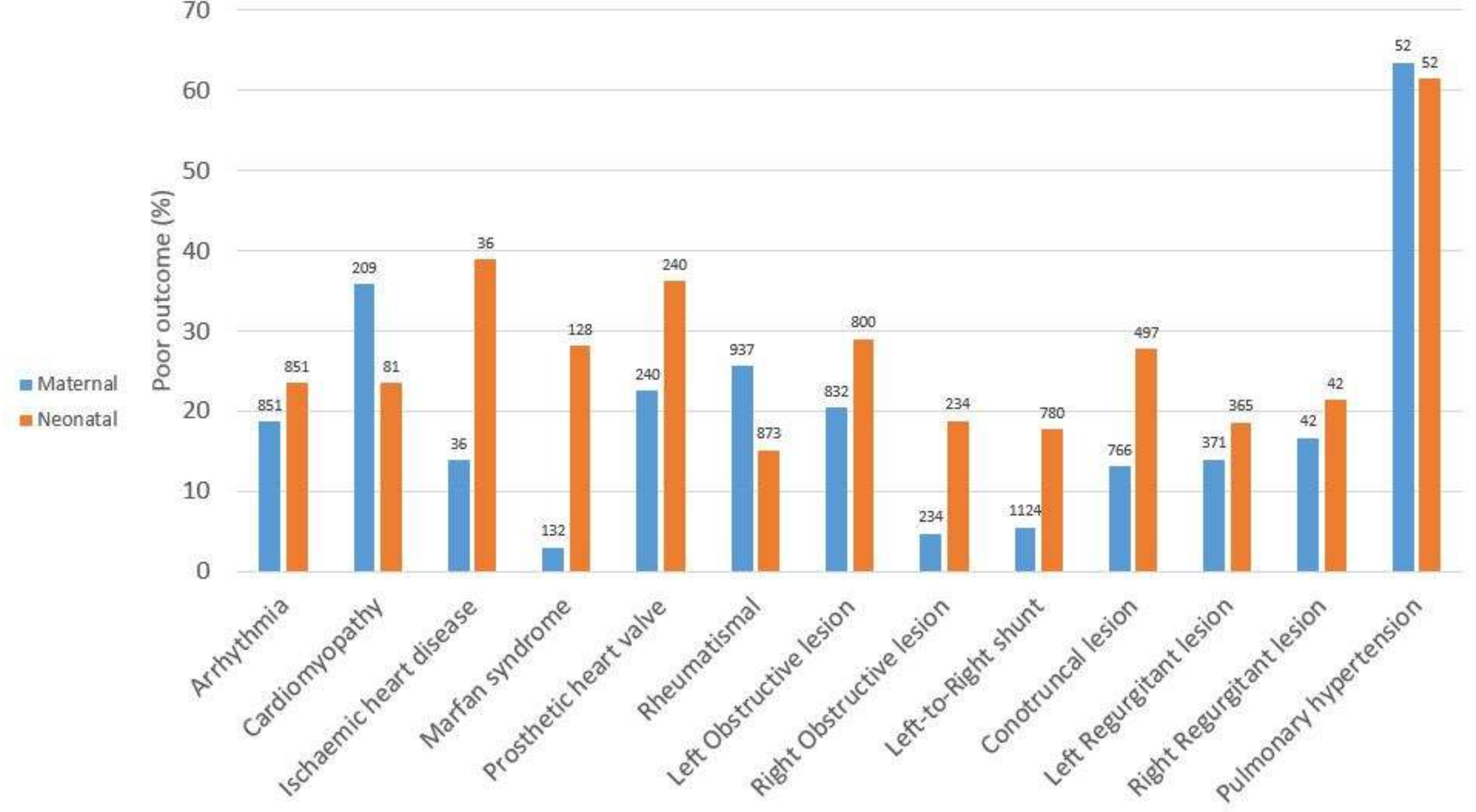

Background and Goal of Study: Management of cardiac diseases, both congenital and acquired, has greatly improved over the last decades. Thus, a vast number of fertile women with heart disease become pregnant each year and cardiac diseases became the second cause of maternal mortality. The aim of this study was to provide an up-to-date estimation of maternal, obstetrical and neonatal outcomes of those high risk pregnancies.

Material and methods: The keywords "(heart disease) AND (pregnancy OR delivery)" were used to search Pubmed, Medline and the Cochrane Database. Studies reporting any outcome of pregnancy in women with pre-existing heart disease, congenital or acquired, were included. Two practitioners independently selected studies, to assess quality of evidence and extract data. The odds ratio associated to every risk factor were calculated separately for the three outcomes. Separate analysis were performed for occidental studies and prospective studies.

\begin{tabular}{lrrrr}
\hline Risk Factor of Maternal Cardiac Event & $\mathbf{N}$ & $\mathbf{p}$ & $\mathbf{i}^{\mathbf{2}}$ \\
\hline BNP>100 pg/ml & 103 & $<.001$ & $0 \%$ \\
Right ventricle dilatation & 109 & $<.001$ & $0 \%$ \\
Oral anticoagulation & 1365 & $<.001$ & $0 \%$ \\
Cardiac history & 2739 & $<.001$ & $84 \%$ \\
NYHA 3-4 & 2663 & $<.001$ & $73 \%$ \\
Low LVEF & 2031 & $<.001$ & $62 \%$ \\
Cardiac medication & 1514 & $<.001$ & $63 \%$ \\
Systemic right ventricle & 1448 & $<.001$ & $0 \%$ \\
Cyanosis & 273 & $<.001$ & $76 \%$ \\
Pacemaker & 1391 & $<.001$ & $0 \%$ \\
Low RVEF & 117 & .007 & $0 \%$ \\
Left heart obstruction & 2511 & $<.001$ & $44 \%$ \\
Pulmonary hypertension & 1402 & $<.001$ & $73 \%$ \\
Right regurgitant & 1584 & $<.001$ & $0 \%$ \\
Left regurgitant & 1365 & .009 & $0 \%$
\end{tabular}

\section{Modified WHO I}

Marfan syndrome

Right Heart Obstruction

Left-to-Right Shunt

Modified WHO 2

Arrhythmias

Ischemic Heart Diseases

Left Heart Obstructions

Left Regurgitant

Right Regurgitant

Conotruncus lesion

\section{Modified WHO 3}

Prosthetic Heart Valve

Cardiomyopathies

Any condition from Modified WHO 2 class with risk factor

\section{WHO 4}

Pulmonary hypertension

Any condition from Modified WHO 3 class with risk factor
Results and Discussion: 59 studies reporting | 4407 pregnancies were included, including 8 prospective studies. Maternal mortality and maternal, obstetrical and neonatal complications were higher than in the general population. Their incidences depended on the baseline cardiac condition. The meta-analysis confirmed that Eisenmenger syndrome and cardiomyopathies were associated with the highest complications rates $(63.5 \% 95 \% \mathrm{Cl}$ [50.5-76.5] and $35.9 \%$ $95 \% \mathrm{Cl}[29.4-42.4]$ respectively). The present literature review gives an updated estimation of the respective weight of all known risk factors of maternal cardiac complication.

and proposes a new stratification of maternal cardiovascular risk, based on the World Health Organization classification. Lastly, this study highlighted two periods especially at risk of maternal cardiac decompensation: the third trimester and the post-partum period.

Conclusions: Risk estimation prior to pregnancy seems achievable, however no strong per pregnancy predictors of decompensation were found. 